Research Article

\title{
Analysis and Stability Control of a Novel 5D Hyperchaotic System
}

\author{
Hong Niu* \\ College of Electronic Information and Automation, Tianjin University of Science \& Technology, China
}

\section{ARTICLE INFO}

\section{Article History}

Received 25 October 2020

Accepted 26 August 2021

Keywords

Novel 5D hyperchaotic system hyperchaos control

Lyapunov stability theory global asymptotic stability

\begin{abstract}
In this paper, a novel Five-Dimensional (5D) autonomous hyperchaotic system is presented, and the characteristics of the 5D system are given in brief. For stability control of the 5D hyperchaotic system, a linear feedback controller and its simplification are designed via the Lyapunov stability theory, so that the 5D system is no longer hyperchaotic but globally asymptotically converges to the equilibrium point at the origin. The numerical simulation results are given to illustrate the feasibility and effectiveness of the method.
\end{abstract}

(C) 2021 The Author. Published by Atlantis Press International B.V. This is an open access article distributed under the CC BY-NC 4.0 license (http://creativecommons.org/licenses/by-nc/4.0/).

\section{INTRODUCTION}

Hyperchaos was first proposed by Otto Rössler in 1979 [1]. Since then, many novel hyperchaotic systems have been formulated. To obtain hyperchaos, the system need to satisfy the following two important requisites. Firstly, the minimal dimension of the phase space that embeds a hyperchaotic attractor should be at least four, which requires the minimum number of coupled first-order autonomous ordinary differential equations to be four. Secondly, the number of terms in the coupled equations giving rise to instability should be at least two, of which at least one should have a nonlinear function [2]. Therefore, hyperchaos is much more complicated than chaos, and it has greater engineering significance and application prospect in signal processing, secure communication and so on.

In this paper, a novel 5D hyperchaotic system, which has been introduced in Wei and Niu [3], is reviewed. Stability control of the 5D system would be discussed, and some simulation results would be given to demonstrate the validity of the designed linear feedback controllers.

\section{THE NOVEL 5D HYPERCHAOTIC SYSTEM}

The dynamic equations of the novel 5D hyperchaotic system are formulated as

$$
\begin{aligned}
& \dot{x}=a(y-x), \\
& \dot{y}=(c-a) x+c y+w-x z, \\
& \dot{z}=-b z+x y, \\
& \dot{v}=m w, \\
& \dot{w}=-y-h v,
\end{aligned}
$$

"Email:spots@163.com; www.tust.edu.cn where $x, y, z, v, w \in R$ are state variables, and $a=23, b=3, c=18$, $m=12$ and $h=4$ [3].

Let the initial values of the $5 \mathrm{D}$ system $(1)$ be $\left(x_{0}, y_{0}, z_{0}, v_{0}, w_{0}\right)=$ $(1,1,1,1,1)$, then the Lyapunov exponents respectively are $\lambda_{1}=$ $0.8732>0, \lambda_{2}=0.1282>0, \lambda_{3}=-0.0013 \approx 0, \lambda_{4}=-0.5770<0$ and $\lambda_{5}=-8.4231<0$. It indicates that the $5 \mathrm{D}$ system ( 1$)$ is hyperchaotic. The attractors of the 5D hyperchaotic system (1) are shown in Figure 1.

\section{HYPERCHAOS CONTROL OF THE 5D SYSTEM}

\subsection{Formulation of the Controlled System}

The controlled system is represented as

$$
\begin{aligned}
& \dot{x}=a(y-x)+u_{c 1}, \\
& \dot{y}=(c-a) x+c y+w-x z+u_{c 2}, \\
& \dot{z}=-b z+x y+u_{c 3}, \\
& \dot{v}=m w+u_{c 4}, \\
& \dot{w}=-y-h v+u_{c 5},
\end{aligned}
$$

where

$$
\begin{aligned}
\boldsymbol{u}_{c} & =\left[\begin{array}{lllll}
u_{c 1} & u_{c 2} & u_{c 3} & u_{c 4} & u_{c 5}
\end{array}\right]^{\mathrm{T}} \\
& =\left[\begin{array}{lllll}
-k_{1} x & -k_{2} y & -k_{3} z & -k_{4} v & -k_{5} w
\end{array}\right]^{\mathrm{T}},
\end{aligned}
$$

and $k_{1}, k_{2}, k_{3}, k_{4}, k_{5} \geq 0$.

\subsection{Design of the Linear Feedback Controller}

Theorem 1. Let $\boldsymbol{x}=\mathbf{0}$ be an equilibrium point for $\dot{\boldsymbol{x}}=\boldsymbol{f}(\boldsymbol{x})$, where $f: D \rightarrow R^{n}$ is a locally Lipschitz map from a domain $D \subset R^{n}$ into $R^{n}$. Let $V: R^{n} \rightarrow R$ be a continuously differentiable function such that 
(a1)

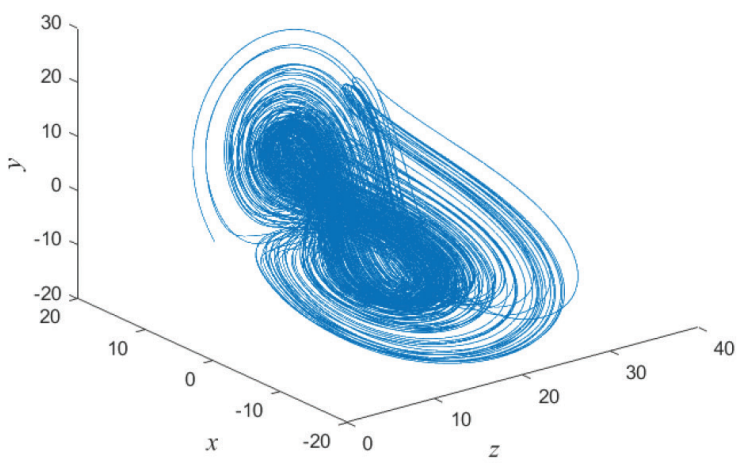

(a3)

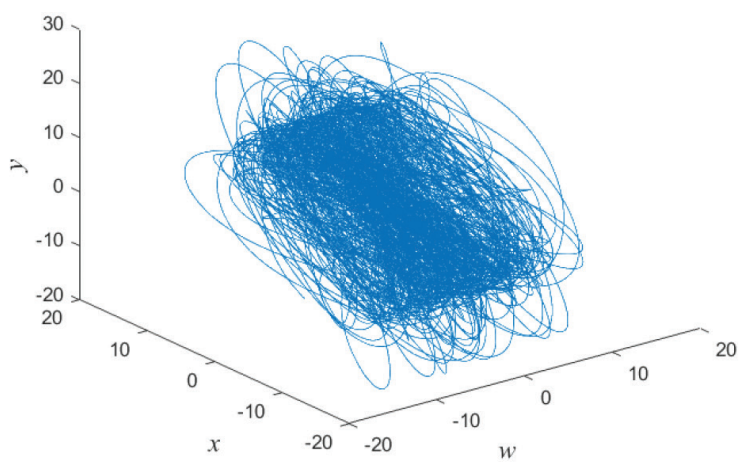

(a5)

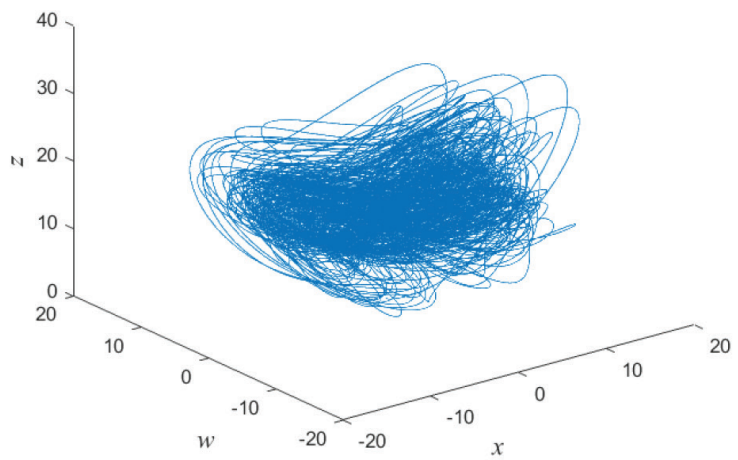

(a2)

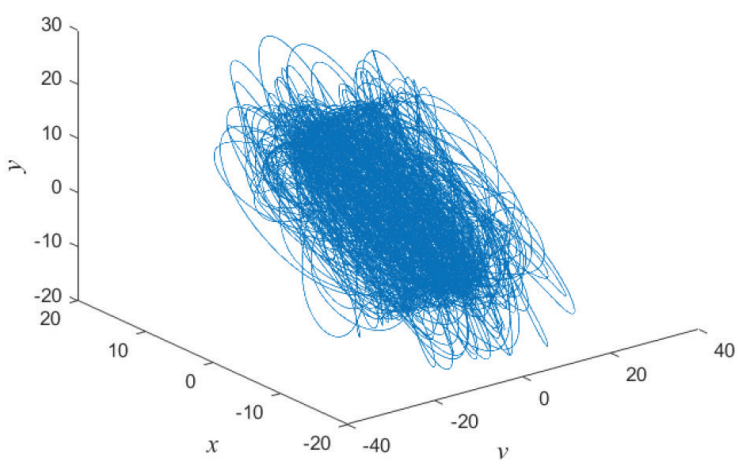

(a4)

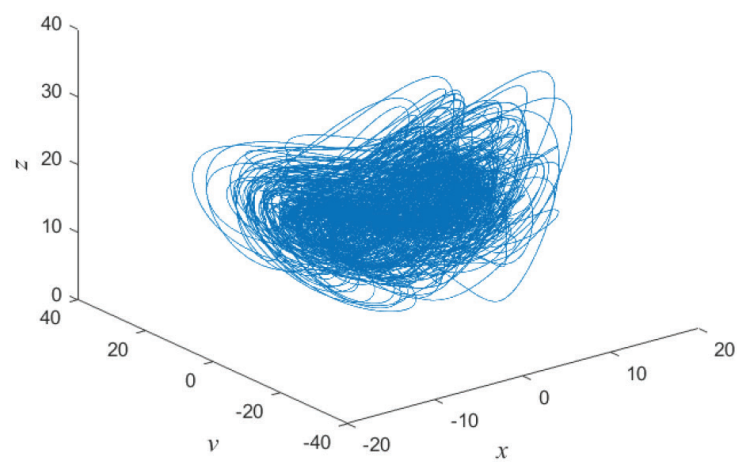

(a6)

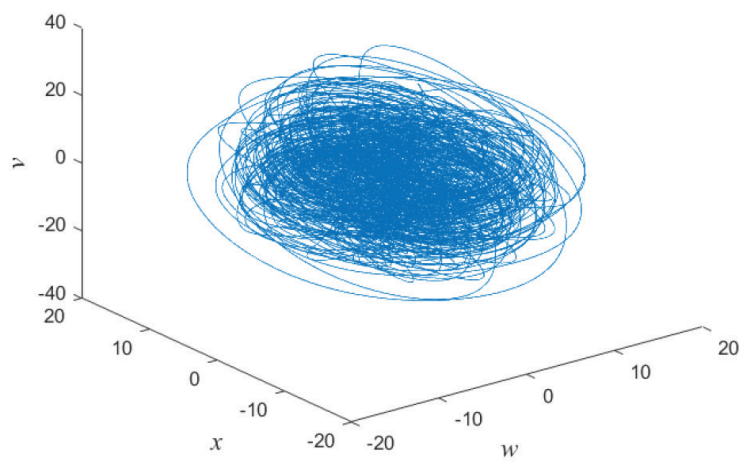

Figure 1 Attractors of the 5D hyperchaotic system: (a1) $z-x-y$; (a2) $v-x-y$; (a3) $w-x-y$; (a4) $x-v-z$; (a5) $x-w-z$; (a6) $w-x-v$.

$$
\begin{aligned}
& V(\mathbf{0})=0 \text { and } V(\boldsymbol{x})>0, \quad \forall \boldsymbol{x} \neq \mathbf{0} \\
& \|\boldsymbol{x}\| \rightarrow \infty \Rightarrow V(\boldsymbol{x}) \rightarrow \infty \\
& \dot{V}(\boldsymbol{x})<0, \quad \forall \boldsymbol{x} \neq \mathbf{0}
\end{aligned}
$$

then $\boldsymbol{x}=\mathbf{0}$ is globally asymptotically stable [4].

From Theorem 1, take a continuously differentiable function

$$
V=\frac{1}{2}\left(x^{2}+y^{2}+z^{2}+\frac{h}{m} v^{2}+w^{2}\right)
$$

as a Lyapunov function candidate for the controlled system (2). Then, the derivative $\dot{V}$ is given by

$$
\begin{aligned}
\dot{V}= & x \dot{x}+y \dot{y}+z \dot{z}+\frac{h}{m} v \dot{v}+w \dot{w} \\
= & -\left(k_{1}+a\right) x^{2}+c x y-\left(k_{2}-c\right) y^{2} \\
& -\left(k_{3}+b\right) z^{2}-k_{4} \frac{h}{m} v^{2}-k_{5} w^{2} \\
\leq & -\left(k_{1}+a-\frac{c}{2}\right) x^{2}-\left(k_{2}-\frac{3}{2} c\right) y^{2} \\
& -\left(k_{3}+b\right) z^{2}-k_{4} \frac{h}{m} v^{2}-k_{5} w^{2} .
\end{aligned}
$$




$$
\begin{array}{rll}
k_{1}+a-\frac{c}{2}>0, & k_{1}>\frac{c}{2}-a, & \\
k_{2}-\frac{3}{2} c>0, & & k_{1}=0, \\
k_{3}+b>0, & k_{2}>\frac{3}{2} c, & \\
k_{2} & =30, \\
k_{3}>-b, & k_{3}=0, \\
k_{4} \frac{h}{m}>0, & k_{4}>0, & k_{4}=1, \\
k_{5}>0, & k_{5}>0,
\end{array}
$$

From Theorem 1, the controlled system (2) is globally asymptotically stable at the origin. Thus, the controller $\boldsymbol{u}_{c}$ can be designed as

$$
\begin{aligned}
\boldsymbol{u}_{c} & =\left[\begin{array}{lllll}
u_{c 1} & u_{c 2} & u_{c 3} & u_{c 4} & u_{c 5}
\end{array}\right]^{T} \\
& =\left[\begin{array}{lllll}
0 & -30 y & 0 & -v & -w
\end{array}\right]^{T} .
\end{aligned}
$$

\subsection{Numerical Simulation under the Controller $u_{c}$}

The curves of the state variables of the controlled system (2) before and after adding the controller $\boldsymbol{u}$ are shown in Figures 2 and 3 respectively. Comparing Figure 3 with Figure 2, it can be found that

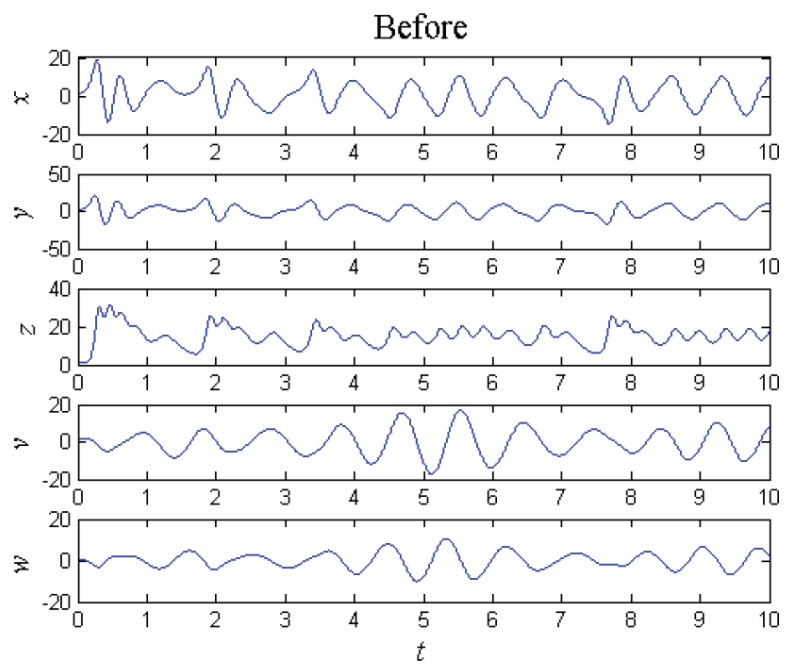

Figure 2 Curves of the state variables before adding $\boldsymbol{u}_{c}$.

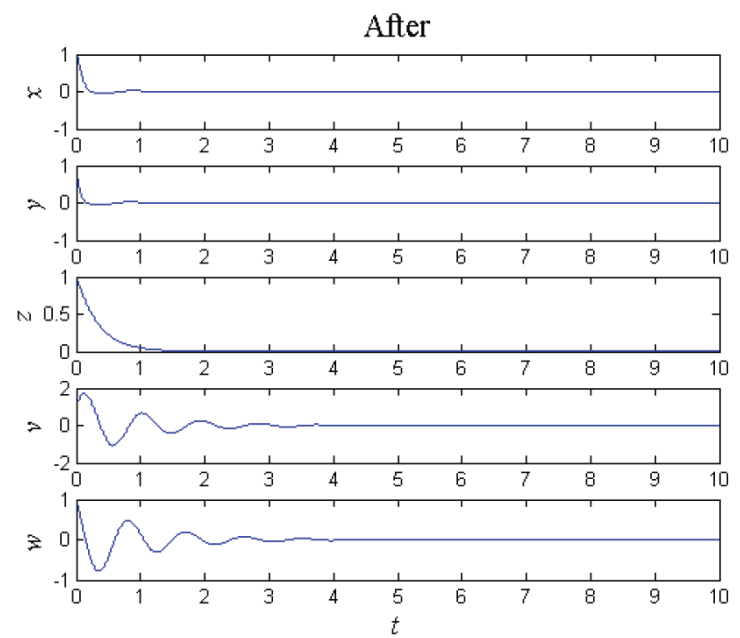

Figure 3 Curves of the state variables after adding $\boldsymbol{u}_{c}$. the state variables $x, y, z, v$ and $w$ converge to zero asymptotically and rapidly. Meanwhile, the Lyapunov exponents of the controlled system (2) are $\lambda c_{1}=-1.0321, \lambda c_{2}=-1.0327, \lambda c_{3}=-3.0000, \lambda c_{4}=$ -17.4666 and $\lambda c_{5}=-17.4687$, which are all negative. It implies that the controlled system (2) is no longer hyperchaotic but asymptotically stable at the origin. It illustrates that the linear feedback controller $\boldsymbol{u}_{c}$ is feasible and effective for hyperchaos control of the 5D system (2).

\subsection{Simplification of the Controller $u_{c}$}

Corollary 1. Let $\boldsymbol{x}=\mathbf{0}$ be an equilibrium point for $\dot{\boldsymbol{x}}=\boldsymbol{f}(\boldsymbol{x})$, where $f: D \rightarrow R^{n}$ is a locally Lipschitz map from a domain $D \subset R^{n}$ into $R^{n}$. Let $V: R^{n} \rightarrow R$ be a continuously differentiable, radially unbounded, positive definite function such that $\dot{V}(\boldsymbol{x}) \leq 0$ for all $\boldsymbol{x} \in R^{n}$. Let $S=\left\{\boldsymbol{x} \in R^{n} \mid \dot{V}(\boldsymbol{x})=0\right\}$ and suppose that no solution can stay identically in $S$, other than the trivial solution $\boldsymbol{x}(t) \equiv \mathbf{0}$. Then, the origin is globally asymptotically stable [4].

Assume that the minimum number of the feedback variables might be equal to the number of the positive Lyapunov exponents [5]. The 5D hyperchaotic system (1) has two positive Lyapunov exponents, but there are three feedback variables in Equation (5). Still take Equation (3) as a Lyapunov function candidate for the controlled system (2). Now let $k_{4}=0$ and substitute $k_{1}=k_{3}=k_{4}=0$ into Equation (4). Then, the derivative $\dot{V}$ is reduced to

$$
\begin{aligned}
\dot{V} & =x \dot{x}+y \dot{y}+z \dot{z}+\frac{h}{m} v \dot{v}+w \dot{w} \\
& =-a x^{2}+c x y-\left(k_{2}-c\right) y^{2}-b z^{2}-k_{5} w^{2} \\
& \leq-\left(a-\frac{c}{2}\right) x^{2}-\left(k_{2}-\frac{3}{2} c\right) y^{2}-b z^{2}-k_{5} w^{2}
\end{aligned}
$$

For $\dot{V} \leq 0$, the parameters $k_{2}$ and $k_{5}$ should satisfy that

$$
\begin{aligned}
k_{2}-\frac{3}{2} c>0, \\
k_{5}>0,
\end{aligned} \Rightarrow \begin{aligned}
& k_{2}>\frac{3}{2} c, \\
& k_{5}>0,
\end{aligned} \Rightarrow \begin{aligned}
& k_{2}=30, \\
& k_{5}=1 .
\end{aligned}
$$

From Corollary 1, to find $S=\left\{\boldsymbol{x} \in R^{5} \mid \dot{V}(\boldsymbol{x})=0\right\}$, note that

$$
\dot{V}=0 \Rightarrow x=y=z=w=0
$$

Hence, $S=\left\{\boldsymbol{x} \in R^{5} \mid x=y=z=w=0\right\}$.

Let $\boldsymbol{x}(t)$ be a solution that belongs identically to $S=\left\{\boldsymbol{x} \in R^{5} \mid x=y=\right.$ $z=w=0\}$, so that

$$
\begin{aligned}
& x(t)=y(t)=z(t)=w(t) \equiv 0 \\
\Rightarrow & \dot{x}(t)=\dot{y}(t)=\dot{z}(t)=\dot{v}(t)=\dot{w}(t) \equiv 0 \\
\Rightarrow & v(t) \equiv 0
\end{aligned}
$$

Therefore, the only solution that can stay identically in $S=\left\{\boldsymbol{x} \in R^{5} \mid \dot{V}(\boldsymbol{x})=0\right\}$ is the trivial solution $\boldsymbol{x}(t) \equiv \mathbf{0}$. Thus, the origin is globally asymptotically stable. Finally, the controller $\boldsymbol{u}_{c}$ in Equation (5) is simplified as

$$
\begin{aligned}
\boldsymbol{u}_{c s} & =\left[\begin{array}{lllll}
u_{c s 1} & u_{c s 2} & u_{c s 3} & u_{c s 4} & u_{c s 5}
\end{array}\right]^{\mathrm{T}} \\
& =\left[\begin{array}{lllll}
0 & -30 y & 0 & 0 & -w
\end{array}\right]^{\mathrm{T}} .
\end{aligned}
$$



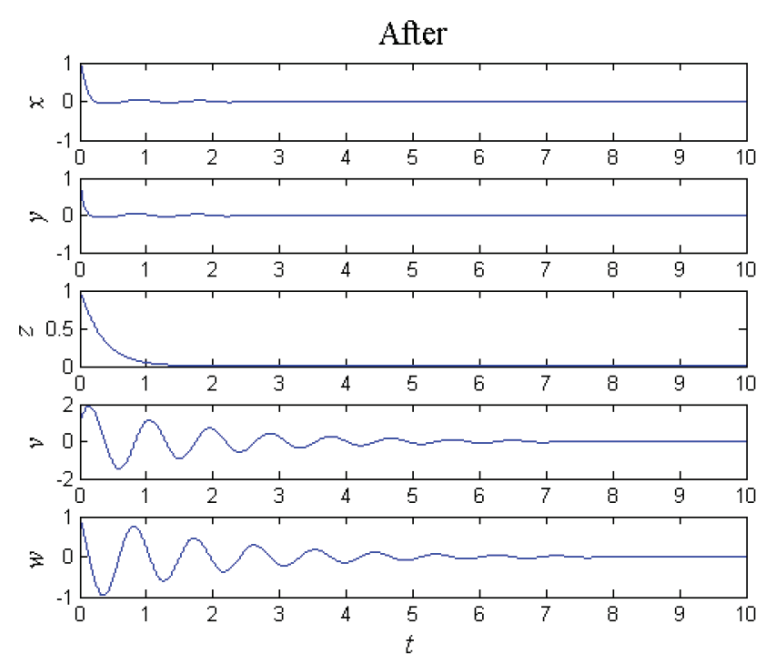

Figure 4 Curves of the state variables after adding $\boldsymbol{u}_{c s}$.

\subsection{Numerical Simulation under the Simplified Controller $u_{c s}$}

Let the initial values still be $\left(x_{0}, y_{0}, z_{0}, v_{0}, w_{0}\right)=(1,1,1,1,1)$, then the curves of the state variables of the controlled system (2) before and after adding the simplified controller $\boldsymbol{u}_{c s}$ are shown in Figures 2 and 4 respectively. Comparing Figure 4 with Figure 2, it can be found that the state variables $x, y, z, v$ and $w$ converge to zero asymptotically and rapidly. Meanwhile, the Lyapunov exponents of the controlled system (2) are $\lambda c s_{1}=-0.53246, \lambda c s_{2}=-0.53248$, $\lambda c s_{3}=-3.0000, \lambda c s_{4}=-17.4664$ and $\lambda c s_{5}=-17.4687$, which are all negative. It implies that the controlled system (2) is no longer hyperchaotic but asymptotically stable at the origin. It illustrates that the simplified linear feedback controller $\boldsymbol{u}_{c s}$ is also feasible and effective for hyperchaos control of the 5D system (2). Furthermore, the simplified controller $\boldsymbol{u}_{c s}$ only has two feedback variables, such that it is easier to implement via circuit than the controller $\boldsymbol{u}_{c}$.

\section{CONCLUSION}

In this paper, a novel 5D hyperchaotic system is reviewed. For hyperchaos control of the 5D system, a linear feedback controller and its simplification are designed via the Lyapunov stability theory. The numerical simulation results demonstrate the validity of the controllers. The study in this paper has some engineering significance.

\section{CONFLICTS OF INTEREST}

The author declares no conflicts of interest.

\section{REFERENCES}

[1] O.E. Rössler, An equation for hyperchaos, Phys. Lett. A 71 (1979), $155-157$.

[2] T. Gao, G. Chen, Z. Chen, S. Cang, The generation and circuit implementation of a new hyper-chaos based upon Lorenz system, Phys. Lett. A 361 (2007), 78-86.

[3] Q. Wei, H. Niu, Analysis and circuit design of a novel 5D hyperchaotic system, Dyn. Syst. Control 8 (2019), 118-128.

[4] I. Karafyllis, A. Chaillet, Lyapunov conditions for uniform asymptotic output stability and a relaxation of Barbălat's lemma, Automatica 132 (2021), 109792.

[5] H. Niu, Analysis and control of a novel 4D chaotic system, Proceedings of the 2017 International Conference on Artificial Life and Robotics, Seagaia Convention Center, Miyazaki, Japan, 2017, pp. 165-168.

\section{AUTHOR INTRODUCTION}

She received the PhD degree in control sci-
ence and engineering from Tianjin University,
China, in 2014. She is currently a lecturer with
the College of Electronic Information and
Automation, Tianjin University of Science \&
Technology, China. Her main research inter-
ests are analysis, control, synchronization and

